ORIGINAL ARTICLE

\title{
Homozygous mutations in LPIN2 are responsible for the syndrome of chronic recurrent multifocal osteomyelitis and congenital dyserythropoietic anaemia (Majeed syndrome)
}

\author{
P J Ferguson, S Chen, M K Tayeh, L Ochoa, S M Leal, A Pelet, A Munnich, S Lyonnet, H A Majeed, \\ H El-Shanti
}

J Med Genet 2005;42:551-557. doi: 10.1136/jmg.2005.030759

See end of article for authors' affiliations

\section{Correspondence to:} Hatem El-Shanti, University of lowa Hospital, 2615 JCP, 200 Hawkins Drive, lowa City, IA 52242, USA; hatem-el-shanti@uiowa. edu

Received 5 January 2005 Revised version received 16 February 2005 Accepted for publication 18 February 2005

\begin{abstract}
Background: Majeed syndrome is an autosomal recessive, autoinflammatory disorder characterised by chronic recurrent multifocal osteomyelitis and congenital dyserythropoietic anaemia. The objectives of this study were to map, identify, and characterise the Majeed syndrome causal gene and to speculate on its function and role in skin and bone inflammation.

Methods: Six individuals with Majeed syndrome from two unrelated families were identified for this study. Homozygosity mapping and parametric linkage analysis were employed for the localisation of the gene responsible for Majeed syndrome. Direct sequencing was utilised for the identification of mutations within the genes contained in the region of linkage. Expression studies and in silico characterisation of the identified causal gene and its protein were carried out.

Results: The phenotype of Majeed syndrome includes inflammation of the bone and skin, recurrent fevers, and dyserythropoietic anaemia. The clinical picture of the six affected individuals is briefly reviewed. The gene was mapped to a $5.5 \mathrm{cM}$ interval $(1.8 \mathrm{Mb})$ on chromosome $18 \mathrm{p}$. Examination of genes in this interval led to the identification of homozygous mutations in LPIN2 in affected individuals from the two families. LPIN2 was found to be expressed in almost all tissues. The function of LPIN2 and its role in inflammation remains unknown.

Conclusions: We conclude that homozygous mutations in LPIN2 result in Majeed syndrome. Understanding the aberrant immune response in this condition will shed light on the aetiology of other inflammatory disorders of multifactorial aetiology including isolated chronic recurrent multifocal osteomyelitis, Sweet syndrome, and psoriasis.
\end{abstract}

M ultifactorial disorders, such as psoriasis and inflammatory bowel disease, result from complex interactions between a number of predisposing factors, including genotypes at one or more loci and a variety of environmental exposures that trigger, accelerate, or exacerbate the disease process. There are several approaches to the identification of aetiologic genetic factors in a complex disease, such as genome-wide linkage and association studies or targeted examination of a specific gene or region. In the latter approach, the specific gene that is targeted in a study is chosen due to its physiologic role, its presence in a chromosomal break point, or its causal role in a Mendelian disorder sharing a similar phenotype. The exploration of the role of a rare Mendelian disorder gene in a more common disorder that shares a phenotype with the Mendelian disorder has proven successful on multiple occasions, such as in WFS1 and deafness, ${ }^{1}$ IRF6 and cleft lip and/or palate, ${ }^{2}$ and rare variations in $A B C A 1, A P O A$, and $\angle C A T$ and low plasma levels of HDL cholesterol.

Chronic recurrent multifocal osteomyelitis (CRMO; MIM 259680) was clinically described in 1970, but it became recognised as a separate clinical entity in $1972 .{ }^{45} \mathrm{CRMO}$ is an autoinflammatory disorder, that is, it is characterised by seemingly unprovoked inflammation in the absence of autoimmunity or infectious causes. It is frequently associated with a cutaneous inflammatory condition such as pustulosis palmoplantaris (PPP), ${ }^{6-8}$ Sweet syndrome, ${ }^{9-11}$ and psoriasis. ${ }^{12-14}$ Although CRMO usually occurs sporadically, several observations point to a genetic component to its aetiology. There are at least three reports of affected siblings with normal parents, suggesting autosomal recessive inheritance. ${ }^{815}{ }^{16} \mathrm{~A}$ published article reports one monozygotic twin pair concordant for CRMO and a second monozygotic twin pair with CRMO diagnosed in one twin and PPP in the other. ${ }^{17}$ In the same article, the authors report a child with CRMO whose father has developed chronic non-infectious osteomyelitis of the sternum as an adult, suggesting autosomal dominant inheritance with variable expression. Also, it has been recently shown through an association study that there is a susceptibility locus for isolated CRMO on chromosome $18 \mathrm{q} 21.3-22 .{ }^{17}$ Furthermore, there is a similar murine disorder (chronic multifocal osteomyelitis), resulting from a recessive mutation in a gene mapped to a region on mouse chromosome 18, which is homologous to the human region containing the CRMO susceptibility locus. ${ }^{18} 19$

The high prevalence of consanguinity among Jordanians makes it possible to identify inbred families with rare recessive disorders that are large enough to independently establish linkage. ${ }^{20-22}$ In these families, individuals with rare recessive traits are likely to have inherited both copies of the mutated gene from a common ancestor and are thus identical by descent (homozygous) not only at the trait locus but also at neighbouring marker loci.

The syndrome of CRMO and congenital dyserythropoietic anaemia (CDA), or Majeed syndrome, is a rare autosomal recessive disorder first described in $1989 .{ }^{23}$ It is composed of three prominent features: CRMO, CDA, and an inflammatory

Abbreviations: CDA, congenital dyserythropoietic anaemia; CRMO, chronic recurrent multifocal osteomyelitis; PPP, pustulosis palmoplantaris 
dermatosis. ${ }^{23-25}$ Majeed syndrome has been described only in two families, although two reported sporadic cases have a similar phenotype. ${ }^{26} 27$ The CRMO starts in infancy and continues relentlessly leading to retarded growth or joint contractures or both..$^{23-25}$ The CDA is variable in severity and characterised by microcytosis, both peripherally and in the bone marrow, which is not typical of any of the well characterised and classified forms of CDA. ${ }^{23-25}$ The neutrophilic dermatosis, Sweet syndrome, is the common reported skin inflammation. ${ }^{23}$ Our group has previously reported six individuals with Majeed syndrome from three sibships from two unrelated families. ${ }^{23-25}$ The gene responsible for this complex syndrome was mapped to a $5.5 \mathrm{cM}$ interval on the short arm of chromosome 18. We then identified that homozygous mutations in LPIN2 are responsible for Majeed syndrome.

\section{METHODS \\ Subjects}

We identified a family from Jordan with four affected individuals. A detailed family history was obtained and a four generation pedigree was constructed (fig 1). The family members who agreed to participate in this study are four affected individuals and seven of their relatives (family A). A second family with two affected individuals in one sibship and first cousin parents was identified, but only the affected individuals participated in the study (family B). Informed consent, approved by a local (Jordanian) institutional review board, was provided by the participants or their legal guardian. We obtained 1064 controls from the HGDP-CEPH Human Genome Diversity Cell Line Panel, ${ }^{28}$ an additional 86 unrelated CEPH controls, and 367 anonymous Jordanian control DNA samples.

\section{Genotyping}

DNA was extracted from whole blood by standard procedures. A genome-wide scan for linkage was performed with the ABI PRISM linkage mapping set of fluorescent microsatellite DNA markers, version 2 (PE Biosystems, Foster City, CA) using affected individuals from family A. To test the regions that showed homozygosity in the screen, typing of all family members with several markers within that region was performed. To refine the region of interest, an additional set of 17 micro-satellite markers was chosen from Marshfield genetic map. ${ }^{29}$ Amplification of these markers was performed by PCR under standard conditions. ${ }^{30}$ The amplified products were analysed on $6 \%$ denaturing polyacrylamide gel (8 M urea) and stained with silver nitrate according to standard protocols. ${ }^{31}$

\section{Statistical analysis}

LOD score analysis was performed using MLINK of the LINKAGE 5.1 computer program package. $^{32}$ Multipoint linkage analysis was performed using SIMWALK2. ${ }^{33}{ }^{34}$ The analysis was carried out assuming autosomal recessive inheritance, full penetrance, and no phenocopies. An assumed disease allele frequency of 0.001 was used in the initial analysis, but was varied from 0.01 to 0.001 to examine the strength of linkage. The allele frequencies for all the markers were assumed to be equal, since the true allele frequency for this population is unknown. We also varied the marker allele frequencies for measurement of the strength of linkage. A standard LOD score of 3 or higher was used for significance. The Marshfield genetic map was used as the reference map for linkage. ${ }^{29}$

Exclusion of candidate genes and mutation detection The region of linkage contains 11 known and predicted genes as obtained from the genome databases (http:/ www.ncbi.nlm.nih.gov; http://genome.ucsc.edu; http:// www.ensembl.org). We prioritised the genes in the region according to their likelihood for producing the phenotype of Majeed syndrome, as follows: TGIF, HEC (KNTC2), EMILIN2, LPIN2, METTL4. The remaining six genes were tissue specific (muscle or neuronal tissue) or the predicted function could not be logically related to the phenotype. The genomic sequences of the candidate genes were obtained from the genome databases and the primer pairs were designed to amplify the exons and the splice sites of the genes using the Primer3 program (http://frodo.wi.mit.edu/cgi-bin/primer3/ primer3_www.cgi). The PCR amplification products were run on an agarose gel, and the bands containing the amplified products were recovered and extracted from the agarose by column purification. The amplified products were sequenced using an automated ABI sequencer with dye terminator chemistry. Sequencing was performed for an obligate carrier and an affected individual from family A, an affected individual from family $\mathrm{B}$, and a normal unrelated control. Once a mutation was identified in family A, the same exon was sequenced in all family members to see if it segregated with both the phenotype and haplotype. We then screened the control populations by both SSCP analysis and sequencing of suspicious bands. For family B, we sequenced the exon that carried the mutation in the other affected individual and developed an SSCP assay to screen the control populations for that mutation. The sequences of the primers used for the amplification of the exons and for the SSCP analysis are included in the supplementary material which is available at http://jmedgenet.com/supplemental.

\section{Structure of LPIN2 and its transcript}

The structure of LPIN2 was obtained from the public genomic databases (D87436; NM_014646). Five human overlapping cDNA clones covering the entire cDNA of LPIN2 were obtained (Research Genetics, Invitrogen, Carlsbad, CA) and sequenced for confirmation of the cDNA sequence. These clones were used as positive controls in the expression studies.

\section{Expression analysis}

Pre-made northern blots containing $2 \mu \mathrm{g}$ of poly(A)+RNA per lane from multiple human tissues were obtained commercially (Clontech Laboratories, Palo Alto, CA). Primers were designed to amplify a 348 bp stretch of mRNA or cDNA from exons 18, 19, and 20. The primers were used to generate a probe by amplification of one of the obtained clones and the probe was labelled radioactively by random priming (Amersham Pharmacia Biotech, Piscataway, NJ). The probe was hybridised overnight in hybridisation buffer at $42^{\circ} \mathrm{C}$. The blot was washed three times with $2 \times$ SSC:0.1\% SDS for 15 min each wash. The blot was then exposed to Kodak XOmat film at $-80^{\circ} \mathrm{C}$. The first strand cDNA was obtained commercially (Invitrogen) and the same primers were used in a standard PCR reaction.

\section{RESULTS}

\section{Clinical picture}

Our group has identified two Jordanian families with Majeed syndrome. Family A had four affected individuals from two sibships (IV-6, IV-8, IV-10, IV-11; fig 1) and family B had two affected siblings (B-1, B-2). All six affected individuals from both families had: (i) CRMO (classic radiographs, multifocal isotope uptake on bone scan, prolonged course, and no response to i.v. antibiotic treatment) that was clinically manifest as recurrent bone and joint pain, (ii) CDA (anaemia with dyserythropoiesis, hypochromia, and microcytosis evident on both blood smear and bone marrow aspirate examination), (iii) recurrent fevers (occurring every 2-4 weeks, 


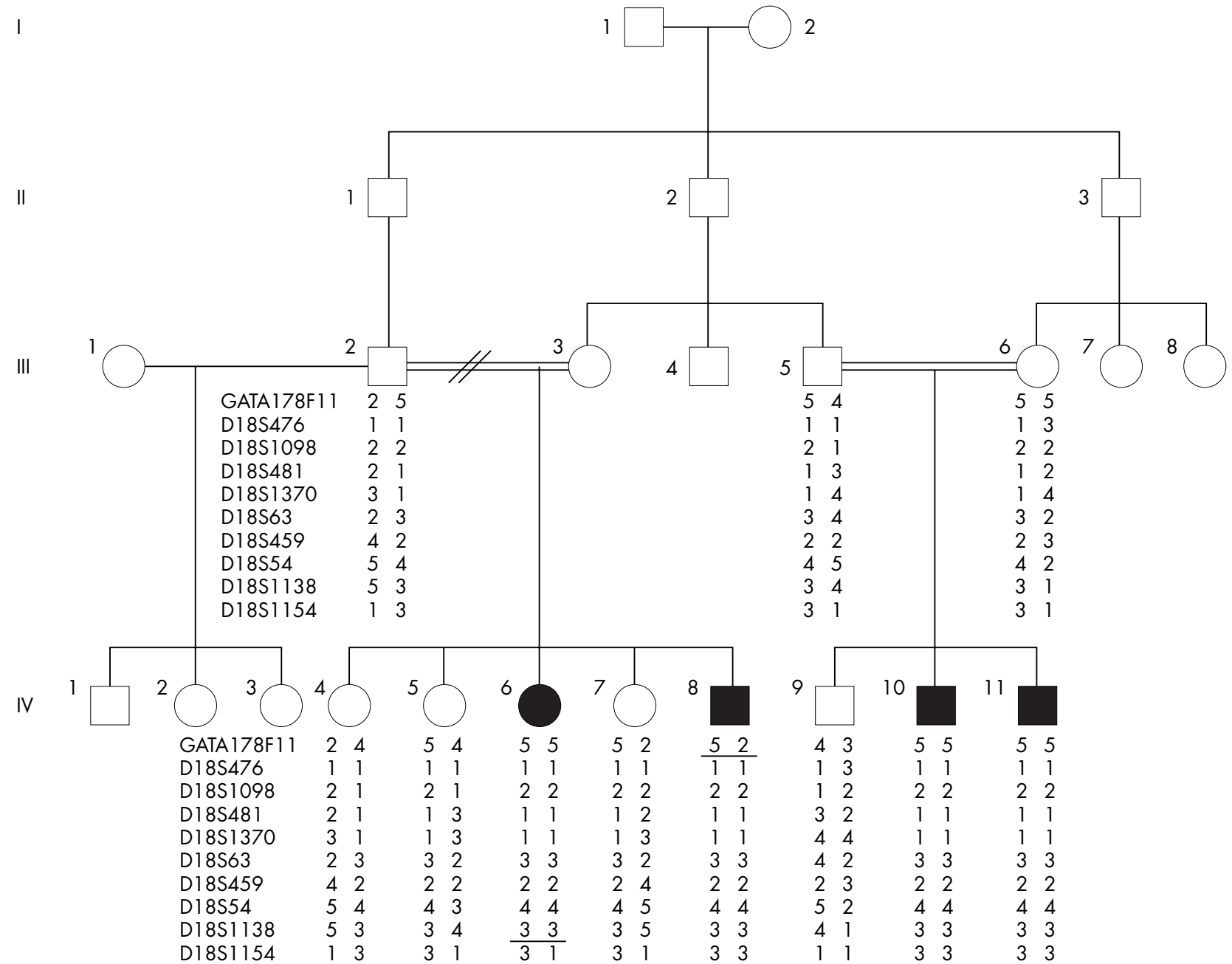

Figure 1 Pedigree of family A showing the haplotypes for 10 microsatellite markers within the region of linkage for all family members and showing the two cross over events.

lasting 3-4 days/episode), and (iv) growth failure. The phenotype was more severe in family $\mathrm{B}$, presenting with higher fevers, more extensive osteomyelitis, and severe transfusion dependent anaemia (table 1 ). ${ }^{24}$

Cutaneous inflammation is also seen in Majeed syndrome. In family A, Sweet syndrome was definitively diagnosed in two affected individuals (IV-10 and IV-11), while two other affected individuals had a history of rash that was consistent with Sweet syndrome (IV-6 and IV-8). The two affected individual from family B did not have Sweet syndrome, but one of them (B-1) had a history of cutaneous pustulosis. One obligate carrier (III-5) had severe psoriasis and III-3 was reported to have psoriasis as well but was not examined. One affected individual from family B (B-1), evaluated at the age of 21 years, had contractures of upper and lower extremity joints, no sexual development, and maxillary bone hyperplasia. The other affected individual from family B (B-2) was ascertained at the age of 2.5 years and had not yet developed contractures or any skin manifestation. The clinical picture of the six affected individuals is summarised in table 1 .

Table 1 Summary of the clinical features of the six affected individuals

\begin{tabular}{|c|c|c|c|c|c|c|}
\hline Clinical feature & A-IV-6 & A-IV-8 & A-IV-10 & A-IV-11 & B-1 & B-2 \\
\hline \multicolumn{7}{|l|}{ CRMO } \\
\hline Bone and joint pain & + & + & + & + & + & + \\
\hline Fever & Mild & Mild & Mild & Mild & High & High \\
\hline Radioisotope scan & + & + & + & + & + & + \\
\hline \multicolumn{7}{|l|}{ CDA } \\
\hline Microcytosis & + & + & + & + & + & + \\
\hline Blood transfusion & No & No & No & No & Frequent & Frequent \\
\hline $\mathrm{Hb}$ electrophoresis & Normal & Normal & Normal & Normal & Normal & Normal \\
\hline \multicolumn{7}{|l|}{ Skin } \\
\hline $\begin{array}{l}\text { Sweet syndrome } \\
\text { Other }\end{array}$ & Yes & Yes & By report & Yes & $\begin{array}{l}\text { No } \\
\text { Pustulosis }\end{array}$ & No \\
\hline Relative with psoriasis & Yes & Yes & Yes & Yes & NA & NA \\
\hline
\end{tabular}




\section{Linkage analysis}

The genome-wide scan showed four regions of homozygosity and all but one were excluded by genotyping all family members for markers within the region of homozygosity. The region of interest, on the short arm of chromosome 18, was refined using 17 microsatellite markers. A maximum twopoint LOD score of 3.48 was obtained for marker D18S1 138 at a recombination fraction $(\theta)=0$ with recombination events at markers GATA178F11 and D18S1154, both marking the region of linkage (table 2). Multipoint linkage analysis produced a maximum LOD score of 4.4 (results not shown). The haplotypes for 10 different markers in the region of linkage were constructed for all the participating family members (fig 1). The minimal region of homozygosity in the affected individuals was $5.5 \mathrm{cM}$ between the microsatellite markers GATA178F11 from the telomeric end and D18S1154 from the centromeric end. ${ }^{29}$ The two affected individuals from family B were homozygous for the 10 markers but for different alleles and so there was no common haplotype (results not shown).

\section{Exclusion of candidate genes}

The markers that define the Majeed syndrome critical region span about $5.5 \mathrm{cM}$ (genetic distance), which is about $1.8 \mathrm{Mb}$ (physical distance) and contains 11 known and predicted genes. LPIN2, which was identified due to a lipin domain it shares with LPIN1, was the fourth on our list of candidate genes. No mutations were identified by sequence analysis in the genes TGIF, KNTC2, and EMILIN2, but two mutations were found in LPIN2.

\section{Mutation detection}

A missense mutation c.2201C $\rightarrow$ T $($ S734L) was identified, which segregates with the phenotype in family A (fig 2). The mutation replaces a polar serine with a non-polar leucine and was not found in 1064 controls obtained from the HGDPCEPH Human Genome Diversity Cell Line Panel or in 86 unrelated CEPH controls (a total of 1150 individuals and 2300 chromosomes). However, this mutation has a frequency of 0.005 (four heterozygotes in 734 chromosomes) in 367 unrelated ethnically matched (Jordanian) controls. The serine at 734 is conserved in human and mouse lipin proteins, and is also conserved in chimpanzee, mouse, rat, chicken, frog, blowfish, mosquito, honey bee, fruit fly, and the nematode Caenorhabditis elegans (fig 3). In family B, we identified a frame shift mutation, c.540-541delAT, which changes the amino acid in position 180 from threonine to proline to be followed by a stop codon in position 181 (fig 2). This mutation was not identified in any individual from the three groups of controls (a total 1517 individuals and 3034 chromosomes).

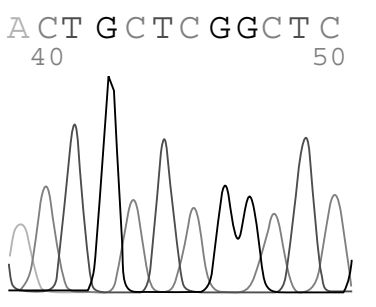

Normal
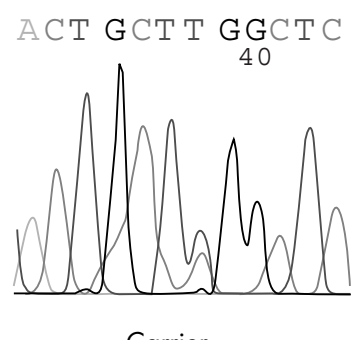

Carrier
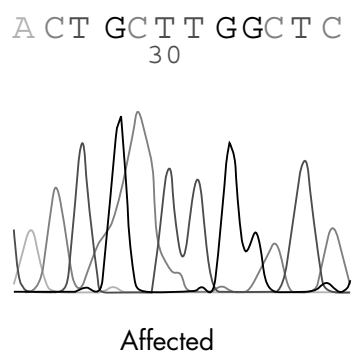

CACA T T G A 290

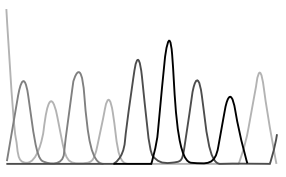

Normal

AT

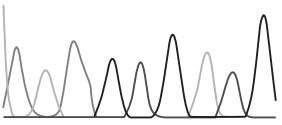

Affected
Figure 2 (A) The sequence of exon 17 of LPIN2 in a normal control, a carrier, and in an affected individual from family A showing the c. $2201 \mathrm{C} \rightarrow \mathrm{T}$ (S734L) mutation. (B) The sequence of exon 4 in a normal control and in an affected individual from family B showing the c.540541 delAT frame shift mutation. The arrow is pointing to the homozygous deletion in the affected individual.

\section{Structure of LPIN2 and its transcript}

LPIN2 (lipin 2), previously called KIAA0249, ${ }^{35}$ consists of 20 exons spanning about $95 \mathrm{~kb}$ and producing a $6228 \mathrm{bp}$ mRNA transcript. The coding sequence is 2691 bp producing an 896 amino acid protein. The translation initiation codon is in exon 2 and the stop codon is at the beginning of exon 20. All of exon 1 and most of exon 20 are untranslated, representing the $5^{\prime}$ and $3^{\prime}$ untranslated regions, respectively. The lipin 2 protein potentially localises to the nucleus as it has a putative nuclear localisation signal. Lipin proteins constitute a novel family of nuclear proteins containing at least three members

Table 2 Linkage analysis results and LOD scores

\begin{tabular}{|c|c|c|c|c|c|c|c|c|c|c|c|}
\hline \multirow[b]{2}{*}{ Marker } & \multirow[b]{2}{*}{ Name } & \multirow[b]{2}{*}{ cM } & \multirow{2}{*}{$\begin{array}{l}\text { Base pairs } \\
\text { starts }\end{array}$} & \multirow{2}{*}{$\begin{array}{l}\text { Base pairs } \\
\text { ends }\end{array}$} & \multicolumn{7}{|c|}{ Recombination fraction $(\theta)$} \\
\hline & & & & & 0.0 & 0.01 & 0.05 & 0.10 & 0.20 & 0.30 & 0.40 \\
\hline & GATA178F11 & 2.84 & 2113098 & 2113470 & $-\infty$ & 1.29 & 1.74 & 1.72 & 1.37 & 0.90 & 0.42 \\
\hline D18S476 & AFM299YF1 & 2.84 & 2167796 & 2168139 & 1.26 & 1.23 & 1.10 & 0.94 & 0.64 & 0.36 & 0.14 \\
\hline D18S1098 & AFMA175YE9 & 5.58 & 2859597 & 2859941 & 1.68 & 1.64 & 1.5 & 1.32 & 0.96 & 0.60 & 0.26 \\
\hline D18S481 & AFM321XC9 & 6.94 & 3056133 & 3056372 & 3.28 & 3.21 & 2.92 & 2.56 & 1.82 & 1.10 & 0.46 \\
\hline D18S1370 & ATA45G06 & 6.94 & 3147651 & 3147789 & 3.07 & 3.00 & 2.73 & 2.38 & 1.67 & 0.99 & 0.39 \\
\hline D18S63 & AFM205TD6 & 8.3 & 3428520 & 3428862 & 2.72 & 2.66 & 2.41 & 2.09 & 1.46 & 0.86 & 0.34 \\
\hline D18S459 & AFM238YD8 & 8.3 & 3524819 & 3525147 & 1.95 & 1.91 & 1.74 & 1.53 & 1.08 & 0.65 & 0.28 \\
\hline D18S54 & AFM080XD7 & 8.3 & 3649285 & 3649553 & 3.41 & 3.33 & 3.04 & 2.67 & 1.91 & 1.16 & 0.49 \\
\hline D18S1138 & AFMB360YH1 & 8.3 & 3662022 & 3662357 & 3.48 & 3.41 & 3.12 & 2.74 & 1.98 & 1.22 & 0.54 \\
\hline D18S1154 & AFMA056YE1 & 8.3 & 3882012 & 3882371 & $-\infty$ & 0.88 & 1.33 & 1.31 & 0.97 & 0.54 & 0.17 \\
\hline
\end{tabular}




$H$ sapiens
$P$ troglodytes
$M$ musculus
$R$ norvegicus
$G$ gallus
$X$ laevis
$T$ nigroviridis
A gambiae
A mellifera
$D$ melanogaster
C elegans

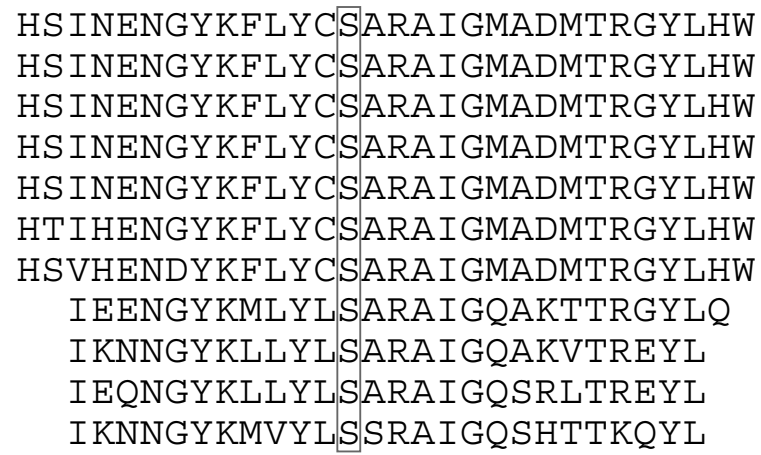

HS INENGYKFLYCSARAI GMADMTRGYLHW

HS INENGYKFLYCSARA I GMADMTRGYLHW

HS INENGYKFLYCSARA I GMADMTRGYLHW

HS INENGYKFLYCSARAIGMADMTRGYLHW

HS INENGYKFLYCSARA I GMADMTRGYLHW

HTIHENGYKFLYCSARAI GMADMTRGYLHW

IEENGYKMLYI SARA IGQAKTTRGYLO

I KNNGYKLLYLSARA I GQAKVTREYL I KNNGYKMVYLSSRAIGQSHTTKQYL

Figure 3 The serine of the S734L mutation is conserved across species. The figure shows the conservation across chimpanzee, mouse, Norway rat, red jungle fowl, African clawed frog, blowfish, mosquito, honey bee, fruit fly, and the nematode $C$ elegans.

in mammalian species and paralogs in distantly related organisms from human to yeast. ${ }^{36}$

\section{Expression analysis}

Global expression of LPIN2 was determined by probing a multiple human tissue northern blot that contains a variety of adult tissues. This experiment determined that LPIN2 is expressed in liver, lung, kidney, placenta, spleen, thymus, lymph node, prostate, testes, small intestine, and colon. It is not expressed in skeletal muscle or in colon that is stripped from its mucosa. There is only one identifiable transcript with a size of about $6 \mathrm{~kb}$. First strand cDNA from multiple human tissues were obtained and expression was examined using primers designed to amplify a $348 \mathrm{bp}$ stretch of cDNA. The expression pattern was similar to the results of the northern blot analysis (fig 4).

\section{DISCUSSION}

Majeed syndrome is composed of three prominent clinical components. The first is CRMO which, unlike isolated CRMO, has an earlier age at onset, more frequent episodes, shorter and less frequent remissions, and is probably life long. The second is CDA, which is characterised by microcytosis both peripherally and in the bone marrow. It can be variable in severity ranging from a mild, unnoticeable anaemia to a blood transfusion dependent form. The third is an inflammatory dermatosis, which can be Sweet syndrome or pustulosis. From clinical observation, it seems that the skin inflammation may manifest in carriers, although the number is too small to clearly state this fact. We propose that Majeed syndrome is an autoinflammatory disorder and falls into the category of periodic fever syndromes.

Majeed syndrome is an autosomal recessive disorder, since male and female siblings are affected and the parents are normal and consanguineous in the two described families. We identified that homozygous mutations in LPIN2 are responsible for the phenotype in the affected individuals in both families. One mutation, c.540-541delAT, is a frame shift mutation that produces a premature stop codon producing a truncated protein that is 180 amino acids long. Since we do not have DNA samples from other family members and could not test for segregation of the mutation within the family, we elected to screen a large control population. We did not identify the mutation in $1150 \mathrm{CEPH}$ controls or in 367 ethnically matched controls. The second mutation, S734L, is a missense mutation that replaces a highly conserved serine with a leucine and segregates with the phenotype in the family. It was not found in a panel of $1150 \mathrm{CEPH}$ controls of different ethnicities. The mutant allele frequency in the ethnically matched controls was 0.005 . It is possible that this mutation is more prevalent in the Jordanian population due to a founder effect. The mutation frequency in the Jordanian population gives a calculated prevalence of 1 in $\sim 35000$ according to the Hardy-Weinberg equilibrium equation
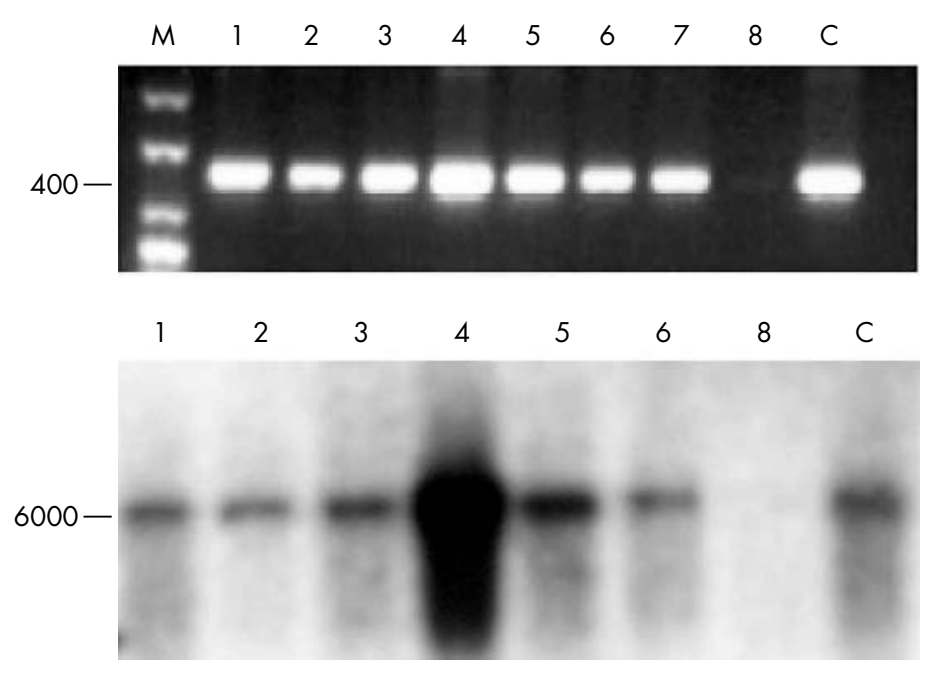

1. Brain

2. Heart

3. Kidney

4. Liver

5. Lung

6. Pancreas

7. Placenta

8. Skeletal muscle

Figure 4 Agarose gel showing the results of PCR amplification of multiple tissue first strand cDNA by LPIN2 cDNA primers (top panel), and the results of the northern blot analysis from similar tissues. $C$, clone; $M$, molecular weight marker. The molecular weight marker for the northern blot $\beta$-actin control is not shown. 
$\left(q=1 / 185\right.$ and $\left.q^{2}=1 / 34225\right)$. Although this figure is higher than the estimated prevalence based on identified cases, we believe that this condition is underdiagnosed due to a lack of awareness of this clinical entity. Based on this data, we conclude that mutations in LPIN2 cause Majeed syndrome.

The clinical description indicates the increased severity of the phenotype in affected individuals from family $\mathrm{B}$ as compared to affected individuals from family A. We speculate that this variability is related to the mutation identified in each family. In family A, the amino acid change probably leads to altered structure and function of the lipin 2 protein. In family $\mathrm{B}$, the truncating mutation most likely leads to complete loss of function, since the protein is only 180 amino acids long.

Little is known about the function of LPIN2. It shares a lipin domain with the human ortholog of murine lpinl which plays a role in fat metabolism. ${ }^{36}$ We placed LPIN2 on our candidate gene list due to considerations regarding its structure and predicted function. Studies of the fission yeast (Schizosaccharomyces pombe) Nedl gene, which belongs to an evolutionary conserved gene family which includes the mammalian LPIN genes, revealed that mutations have a high incidence of chromosome missegregation, aberrantly shaped nuclei, overdeveloped endoplasmic reticulum-like membranes, and increased sensitivity to microtubule destabilising agents. ${ }^{37}$ This description is reminiscent of the dyserythropoiesis seen in Majeed syndrome. In addition, the role of human lipin 2 in fat metabolism is not clear, since LPINI mutations have not been reported in patients with lipodystrophy..$^{38}$ Based on the presence of dyserythropoeisis in this syndrome, we propose that LPIN2 also plays a role in mitosis and its absence or disturbed function leads to abnormal mitosis, especially in rapidly dividing cells such as the RBC lineage in the bone marrow.

We hypothesise that lipin 2 plays a role in the regulation of the innate immune system, and the defect in the protein would lead to increased production of proinflammatory signals. One way that this could occur would be if lipin 2 plays a role in promoting apoptosis in polymorphonuclear leukocytes and its normal function is to curtail the inflammatory milieu. Mutations in LPIN2 will lead to a disturbed function and persistence of inflammation. The assumption would be that there is tissue specific redundancy in the role played by lipin 2 and its related pathway in the down regulation of inflammation, with a lower level of redundancy in skin and bone. We also hypothesise that variations in LPIN2 may play an aetiologic role in psoriasis and related inflammatory skin disorders as it falls within a chromosomal region identified as a psoriasis susceptibility locus. ${ }^{39}$ This hypothesis is further supported by the fact that at least two heterozygotes of LPIN2 mutations and all homozygotes from Majeed syndrome families have psoriasis or another inflammatory dermatosis.

\section{ACKNOWLEDGEMENTS}

The authors are grateful to the families who participated in this study. The authors wish to thank Dr Jeffrey C Murray for kindly providing access to the CEPH controls as well as to laboratory support, and for reviewing the manuscript. The authors are also grateful for the technical advice provided by Dr Brian Schutte.

\section{ELECTRONIC-DATABASE INFORMATION}

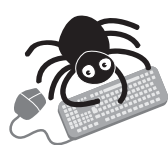

Genome databases mentioned in this paper are to be found at http://www.ncbi.nlm.nih.gov, http:// genome.ucsc.edu, and hitp://www.ensembl.org. The Primer3 program can be found at http://frodo.wi. mit.edu/cgi-bin/primer3/primer3_www.cgi, while primer sequences are available from http:// imedgenet.com/supplemental.

\section{Authors' affiliations}

P J Ferguson, Department of Pediatrics, Division of Hematology/ Oncology/Rheumatology, University of lowa, lowa City, IA, USA S Chen, Department of Pediatrics, University of lowa, lowa City, IA, USA M K Tayeh, Department of Pediatrics, Howard Hughes Medical Institute, University of lowa, lowa City, IA, USA

L Ochoa, Department of Pediatrics, University of lowa, lowa City, IA, USA

S M Leal, Department of Molecular and Human Genetics, Baylor College of Medicine, Houston, TX, USA

A Pelet, Department of Medical Genetics, INSERM U393, Hôpital

Necker-Enfants Malades, Paris, France

A Munnich, Department of Medical Genetics, INSERM U393, Hôpital Necker-Enfants Malades, Paris, France

S Lyonnet, Department of Medical Genetics, INSERM U393, Hôpital

Necker-Enfants Malades, Paris, France

H A Majeed, Department of Pediatrics, University of Jordan, Amman, Jordan

H El-Shanti, Department of Pediatrics, Division of Medical Genetics, University of lowa, lowa City, IA, USA

$\mathrm{H}$ El-Shanti was supported by "Chaire Internationale de Recherche, Blaise Pascal, de l'etat et de la Règion d'lle-de-France" which is managed with further support by the "Fondation de l'Ecole Normale Supèrieure" when the project started (1999-2000). P Ferguson receives funding from the NIH/NICHD Children's Health Research Center Grant, the University of lowa's Department of Pediatrics, and the Roy J. and Lucille A. Carver College of Medicine.

Competing interests: none declared

\section{REFERENCES}

1 Young TL, Ives E, Lynch E, Person R, Snook S, MacLaren L, Cater T, Griffin A, Fernandez B, Lee MK, King MC. Non-syndromic progressive hearing loss DFNA38 is caused by heterozygous missense mutation in the Wolfram syndrome gene WFS1. Hum Mol Genet 2001; 10(22):2509-14.

2 Zucchero TM, Cooper ME, Maher BS, Daack-Hirsch S, Nepomuceno B, Ribeiro L, Caprau D, Christensen K, Suzuki Y, Machida J, Natsume N, Yoshiura K, Vieira AR, Orioli IM, Castilla EE, Moreno L, Arcos-Burgos M Lidral AC, Field LL, Liu YE, Ray A, Goldstein TH, Schultz RE, Shi M, Johnson MK, Kondo S, Schutte BC, Marazita ML, Murray JC. Interferon regulatory factor 6 (IRF6) gene variants and the risk of isolated cleft lip or palate. N Engl J Med 2004;351(8):769-80.

3 Cohen JC, Kiss RS, Pertsemlidis A, Marcel YL, McPherson R, Hobbs HH. Multiple rare alleles contribute to low plasma levels of $\mathrm{HDL}$ cholesterol. Science 2004;305(5685):869-72.

4 Keipert JA, Campbell PE. Recurrent hyperostosis of the clavicles: an undiagnosed syndrome. Aust Paediatr J 1970:6(1):97-104.

5 Giedion A, Holthusen W, Masel LF, Vischer D. Subacute and chronic "symmetrical" osteomyelitis. Ann Radiol (Paris) 1972;15(3):329-42.

6 Bergdahl K, Bjorksten B, Gustavson KH, Liden S, Probst F. Pustulosis palmoplantaris and its relation to chronic recurrent multifocal osteomyelitis. Dermatologica 1979;159(1):37-45.

7 Job-Deslandre C, Krebs S, Kahan A. Chronic recurrent multifocal osteomyelitis: five-year outcomes in 14 pediatric cases. Joint Bone Spine 2001;68(3):245-51

8 Paller AS, Pachman L, Rich K, Esterly NB, Gonzalez-Crussi F. Pustulosis palmaris et plantaris: its association with chronic recurrent multifocal osteomyelitis. J Am Acad Dermatol 1985;12(5 Pt 2):927-30.

9 Arndt JH. Sweet's syndrome and chronic recurrent multifocal osteomyelitis [letter]. Am J Dis Child 1987;141(7):721.

10 Edwards TC, Stapleton FB, Bond MJ, Barrett FF. Sweet's syndrome with multifocal sterile osteomyelitis. Am J Dis Child 1986;140(8):817-8.

11 Nurre LD, Rabalais GP, Callen JP. Neutrophilic dermatosis-associated sterile chronic multifocal osteomyelitis in pediatric patients: case report and review. Pediatr Dermatol 1999;16(3):214-6.

12 Laxer RM, Shore AD, Manson D, King S, Silverman ED, Wilmot DM. Chronic recurrent multifocal osteomyelitis and psoriasis - a report of a new association and review of related disorders. Semin Arthritis Rheum 1988;17(4):260-70.

13 Prose NS, Fahrner U, Miller CR, Layfield L. Pustular psoriasis with chronic recurrent multifocal osteomyelitis and spontaneous fractures. J Am Acad Dermatol 1994:31(2 Pt 2):376-9.

14 Vittecoq O, Said LA, Michot C, Mejiad O, Thomine JM, Mitrofanoff P, Lechevallier J, Ledosseur P, Gayet A, Lauret P, le Loet X. Evolution of chronic recurrent multifocal osteitis toward spondylarthropathy over the long term. Arthritis Rheum 2000:43(1):109-19.

15 Ben Becher S, Essaddam H, Nahali N, Ben Hamadi F, Mouelhi MH, Hammou A, Hadj Romdhane L, Ben Cheikh M, Boudhina T, Dargouth M. [Recurrent multifocal periostosis in children. Report of a familial form]. Ann Pediatr (Paris) 1991;38(5):345-9.

16 Festen JJ, Kuipers FC, Schaars AH. Multifocal recurrent periostitis responsive to colchicine. Scand J Rheumatol 1985;14(1):8-14.

17 Golla A, Jansson A, Ramser J, Hellebrand H, Zahn R, Meitinger T, Belohradsky BH, Meindl A. Chronic recurrent multifocal osteomyelitis 
(CRMO): evidence for a susceptibility gene located on chromosome 18q21.318q22. Eur J Hum Genet 2002;10(3):217-21.

18 Byrd L, Grossmann M, Potter M, Shen-Ong GL. Chronic multifocal osteomyelitis, a new recessive mutation on chromosome 18 of the mouse. Genomics 1991:11(4):794-8.

19 Hentunen TA, Choi SJ, Boyce BF, Dallas MR, Dallas SL, Shen-Ong GL, Roodman GD. A murine model of inflammatory bone disease. Bone 2000;26(2): 183-8.

20 Khoury SA, Massad D. Consanguineous marriage in Jordan. Am J Med Genet 1992;43(5):769-75.

21 Khoury SA, Massad DF. Consanguinity, fertility, reproductive wastage, infant mortality and congenital malformations in Jordan. Saudi Med J 2000;21(2): 150-4.

22 Al-Salem M, Rawashdeh N. Consanguinity in north Jordan: prevalence and pattern. J Biosoc Sci 1993;25(4):553-6.

23 Majeed HA, Kalaawi M, Mohanty D, Teebi AS, Tunjekar MF, al-Gharbawy F, Majeed SA, al-Gazzar AH. Congenital dyserythropoietic anemia and chronic recurrent multifocal osteomyelitis in three related children and the association with Sweet syndrome in two siblings. J Pediatr 1989;115(5 Pt 1):730-4.

24 Majeed HA, Al-Tarawna M, El-Shanti H, Kamel B, Al-Khalaileh F. The syndrome of chronic recurrent multifocal osteomyelitis and congenital dyserythropoietic anaemia. Report of a new family and a review. Eur J Pediatr 2001; 160(12):705-10.

25 Majeed HA, El-Shanti H, Al-Rimawi H, Al-Masri N. On mice and men: an autosomal recessive syndrome of chronic recurrent multifocal osteomyelitis and congenital dyserythropoietic anemia. J Pediatr 2000;137(3):441-2.

26 Vermylen C, Scheiff JM, Rodhain J, Ninane J, Cornu G. A variant of the congenital dyserythropoietic anaemia type II with structural abnormalities in the granulocytic series. Eur J Pediatr 1986;145(3):232-5.

27 Sakran W, Lumelsky D, Schildkraut V, Smolkin V, Halevy R, Koren A. Chronic recurrent multifocal osteomyelitis in infancy: a case report. Clin Pediatr (Phila) 2003;42(8):741-4.

28 Cann HM, de Toma C, Cazes L, Legrand MF, Morel V, Piouffre L, Bodmer J Bodmer WF, Bonne-Tamir B, Cambon-Thomsen A, Chen Z, Chu J, Carcassi C, Contu L, Du R, Excoffier L, Ferrara GB, Friedlaender JS, Groot H, Gurwitz D, Jenkins T, Herrera RJ, Huang X, Kidd J, Kidd KK, Langaney A, Lin AA, Mehdi SQ, Parham P, Piazza A, Pistillo MP, Qian Y, Shu Q, Xu J, Zhu S,
Weber JL, Greely HT, Feldman MW, Thomas G, Dausset J, Cavalli-Sforza LL. A human genome diversity cell line panel. Science 2002;296(5566):261-2.

29 Broman KW, Murray JC, Sheffield VC, White RL, Weber JL. Comprehensive human genetic maps: individual and sex-specific variation in recombination. Am J Hum Genet 1998;63(3):861-9.

30 El-Shanti H, Murray JC, Semina EV, Beutow KH, Scherpbier T, al-Alami J. Assignment of gene responsible for progressive pseudorheumatoid dysplasia to chromosome 6 and examination of COLIOA 1 as candidate gene. Eur J Hum Genet 1998;6(3):251-6.

31 Bassam BJ, Caetano-Anolles G, Gresshoff PM. Fast and sensitive silver staining of DNA in polyacrylamide gels. Anal Biochem 1991;196(1):80-3.

32 Lathrop GM, Laloue JM, Julier C, Ott J. Multilocus linkage analysis in humans: detection of linkage and estimation of recombination. Am J Hum Genet 1985;37(3):482-98.

33 Sobel E, Lange K. Descent graphs in pedigree analysis: applications to haplotyping, location scores, and marker-sharing statistics. Am J Hum Genet 1996;58(6): 1323-37.

34 Weeks DE, Sobel E, O'Connell JR, Lange K. Computer programs for multilocus haplotyping of general pedigrees. Am J Hum Genet 1995:56(6):1506-7.

35 Nagase T, Seki N, Ishikawa K, Ohira M, Kawarabayasi Y, Ohara O, Tanaka A, Kotani H, Miyajima N, Nomura N. Prediction of the coding sequences of unidentified human genes. VI. The coding sequences of 80 new genes (KIAA0201-KIAA0280) deduced by analysis of cDNA clones from cell line KG-1 and brain. DNA Res 1996;3(5):321-9, 341-54.

36 Peterfy M, Phan J, Xu P, Reue K. Lipodystrophy in the fld mouse results from mutation of a new gene encoding a nuclear protein, lipin. Nat Genet $2001 ; 27(1): 121-4$.

37 Tange $Y$, Hirata A, Niwa $O$. An evolutionarily conserved fission yeast protein, Ned 1, implicated in normal nuclear morphology and chromosome stability, interacts with Dis3, Pim 1/RCC1 and an essential nucleoporin. J Cell Sci 2002; 115(Pt 22):4375-85.

$38 \mathrm{Cao}$ H, Hegele RA. Identification of single-nucleotide polymorphisms in the human LPIN1 gene. J Hum Genet 2002:47(7):370-2.

39 Asumalahti K, Laitinen T, Lahermo P, Suomela S, Itkonen-Vatjus R, Jansen C, Karvonen J, Karvonen SL, Reunala T, Snellman E, Uurasmaa T, SaarialhoKere U, Kere J. Psoriasis susceptibility locus on 18 p revealed by genome scan in Finnish families not associated with PSORS1. J Invest Dermatol 2003;121(4):735-40.

\section{Call for papers}

11 th European Forum on Quality Improvement in Health Care 26-28 April 2006, Prague, Czech Republic

Deadline 30 September 2005.

For further information and to submit online go to: www.quality.bmipg.com 